\title{
STANDARD AND ALTERNATIVE ALGEBRAS WITH COMPLETELY REDUCIBLE DERIVATION ALGEBRAS
}

\author{
ERNEST L. STITZINGER
}

\begin{abstract}
Let $\mathfrak{A}$ be a finite-dimensional standard or alternative algebra over a field of characteristic 0 . A necessary and sufficient condition is found such that the derivation algebra of $\mathfrak{U}$ is completely reducible acting on $\mathfrak{A}$.
\end{abstract}

The following type of result occasionally occurs in the literature: If $\mathfrak{A}$ is an algebra of a particular type over a field of characteristic 0 , then (with possibly a few exceptions) $\mathfrak{A}$ is semisimple if and only if the derivation algebra of $\mathfrak{A}$ is semisimple or 0 . It seems natural to ask if placing other natural conditions on the derivation algebra will lead to characterizations of the algebra. The closest condition to semisimplicity of the derivation algebra is complete reducibility of the derivation algebra acting on $\mathfrak{A}$. We show the following:

THEOREM. Let $\mathfrak{A}$ be a standard or alternative algebra over a field of characteristic 0 . Then the derivation algebra $\mathfrak{D}(\mathfrak{U})$ of $\mathfrak{A}$ acts completely reducibly on $\mathfrak{A}$ if and only if the radical $\mathfrak{N}$ of $\mathfrak{A}$ is contained in the center $\mathfrak{C}$ of $\mathfrak{A}$ and $\mathfrak{N}^{2}=\mathfrak{N} \mathfrak{N}=0$.

The main techniques used are results of Schafer ([8], [10]) on standard and alternative algebras and conditions due to Jacobson for linear Lie algebras to be completely reducible ([5], [6]). All algebras considered here are finite dimensional and the unexplained notation is as in [9].

1. The standard case. A. A. Albert has defined an algebra to be standard if the identities

$$
(x, y, z)+(z, x, y)-(x, z, y)=0
$$

and

$$
(x, y, w z)+(w, y, x z)+(z, y, w x)=0
$$

are satisfied where $(x, y, z)$ denotes the associator $(x, y, z)=(x y) z-x(y z)$. Standard algebras include commutative Jordan algebras as well as

Received by the editors March 8, 1973.

AMS (MOS) subject classifications (1970). Primary 17A30, 17A15.

(c) American Mathematical Society 1974 
associative algebras. Further results in this direction are found in [7] and [10]. We mention in particular that if $\mathfrak{A}$ is a standard algebra of characteristic 0 , then $\mathfrak{U}$ is semisimple with each component of dimension $\neq 3$ over its center if and only if $\mathfrak{D}(\mathfrak{A})$ is semisimple or zero. However we do not use this fact here.

Proof of THE STANDARd CASE. Suppose $\mathfrak{N} \subseteq \mathbb{C}$ and $\mathfrak{N}^{2}=0$. Let $\mathfrak{U}=\mathfrak{N}+\mathfrak{S}$ be a Wedderburn decomposition of $\mathfrak{A}$ (Theorem 4 of [10]). Then $\mathfrak{S}$ is the vector space direct sum

$$
\mathfrak{s}=3+\mathfrak{S}^{\prime}+\mathfrak{B}
$$

where 3 is the center of $\mathfrak{S}, \mathfrak{S}^{\prime}$ is the space spanned by all commutators $[x, y]=x y-y x$ of $\mathfrak{S}$ and $\mathfrak{B}$ is the space spanned by all associators of $\mathfrak{S}$ (Lemma 3 of $[10]$ ). We show that $\mathfrak{D}(\mathfrak{A})=\mathfrak{D}_{1}+\mathfrak{D}_{2}$ where

and

$$
\mathfrak{D}_{1}=\{D \in \mathfrak{D}(\mathfrak{U}) ; D: \mathfrak{S} \rightarrow \mathfrak{S}, D: \mathfrak{N} \rightarrow 0\}
$$

$$
\mathfrak{D}_{2}=\{D \in \mathfrak{D}(\mathfrak{U}) ; D: \mathfrak{S} \rightarrow 0\}
$$

and that $\mathfrak{D}_{1}$ and $\mathfrak{D}_{2}$ act completely reducibly on $\mathfrak{U}$. Since $\mathfrak{D}(\mathfrak{U})$ is then the direct sum of $\mathfrak{D}_{1}$ and $\mathfrak{D}_{2}, \mathfrak{D}(\mathfrak{U})$ will act completely reducibly on $\mathfrak{A}$.

Let $D \in \mathfrak{D}(\mathfrak{A})$. Then $D$ restricted to $\mathfrak{S}$ is a derivation from $\mathfrak{S}$ into $\mathfrak{A}$ and this can be extended to a derivation $\hat{D}$ of $\mathfrak{A}$ where

$$
\hat{D}=R_{x}-L_{x}+\sum\left[L_{y_{i}}, R_{z_{i}}\right]
$$

where $x, y_{i}, z_{i} \in \mathfrak{U}$ by Theorem 6 of [10]. Since $\mathfrak{N} \subseteq \mathbb{C}, x, y_{i}, z_{i}$ may be chosen from $\mathfrak{S}$ so that $\hat{D} \in \mathfrak{D}_{1}$ and $D: \mathfrak{S} \rightarrow \mathfrak{S}$. For the same reason any derivation of $\subseteq$ can be extended to an element of $\mathfrak{D}_{1}$. Therefore, to show that $\mathfrak{D}_{1}$ acts completely reducibly on $\mathfrak{A}$, it suffices to show that $\mathfrak{D}(\mathfrak{S})$ acts this way on $\mathfrak{S}$. Since $\mathfrak{S}$ is semisimple, $\mathfrak{D}(\mathfrak{S}) \subseteq \mathfrak{L}(\mathfrak{S})$ where $\mathfrak{L}(\mathfrak{S})$ is the Lie multiplication algebra of $\mathfrak{S}$ (Theorem 6 of $[\mathbf{1 0}]$ ) and $\mathfrak{L}(\mathfrak{S})$ acts completely reducibly on $\mathfrak{S}$. Furthermore, $\mathfrak{L}(\mathfrak{S})=R(\mathfrak{S})+L(\mathfrak{S})+[L(\mathfrak{S}), R(\mathfrak{S})]$ (Theorem 2 of [10]) where $R(\Im)(L(\Im))$ is the space of right (left) multiplications of $\Im$. Also each derivation of $\Im$ is of the form (4) where $x, y_{i}, z_{i} \in \Im$ since $\subseteq$ is semisimple and hence has an identity. It is now clear that $\mathfrak{L}(\mathfrak{S})=\mathfrak{D}(\mathfrak{S}) \oplus R(\mathfrak{S})$ and that $[R(\mathfrak{S}), \mathfrak{D}(\mathfrak{S})] \subseteq R(\mathfrak{S})$. Now each nilpotent element of $\mathfrak{L}(\mathfrak{S})$ can be embedded in a 3-dimensional simple Lie algebra (Theorem 17, p. 100 of [6]) and the same holds for $\mathfrak{D}(\mathfrak{S})$ by Lemma 8, p. 99 of [6]. Since derivation algebras are almost algebraic (Theorem 16, p. 179 of $[3]), \mathfrak{D}(\mathfrak{S})$ acts completely reducibly on $\mathfrak{S}$ (Theorem 17, p. 100 of [6]). Hence $\mathfrak{D}_{1}$ acts completely reducibly on $\mathfrak{A}$.

We next show that if $D \in \mathfrak{D}(\mathfrak{U})$, then $D$ restricted to $\mathfrak{N}$ commutes with all $R_{a}, a \in \mathcal{Z}$, and conversely any such linear transformation of $\mathfrak{N}$ can be extended to an element of $\mathfrak{D}_{2}$. Since $\mathfrak{N} \subseteq \mathfrak{C}$, we have $\mathfrak{C}=\mathfrak{N}+\mathfrak{3}$ and $\mathfrak{C}$ 
is always a characteristic subspace of $\mathfrak{A}$. Now $D$ restricted to $\mathfrak{C}$ is of the form an inner derivation plus a derivation of $\mathfrak{C}$ which annihilates 3 [4, Theorem 4.3]. Since $\mathbb{C}$ is commutative, the first of these derivations is 0 . Now if $n \in \mathfrak{N}, c \in \mathfrak{C}$, then

$$
(n c) D=(n D) c+n(c D)=(n D) c
$$

since $c D \in \mathfrak{N}$ and $\mathfrak{N}^{2}=0$. That is, on $\mathfrak{N}$,

$$
R_{c} D=D R_{c}
$$

for all $c \in \mathfrak{C}$. Also any linear transformation $D$ of $\mathfrak{N}$ which satisfies (5) can be extended to a derivation $E$ of $\mathfrak{C}$ such that $z E=0$ for all $z \in \mathcal{Z}$. In the same manner as in the proof of Theorem 7 of [10], each of these latter derivations of $\mathfrak{C}$ can be extended to a derivation of $\mathfrak{A}$ which annihilates $\mathfrak{S}^{\prime}+\mathfrak{B}$. We now see that $\mathfrak{D}(\mathfrak{H})=\mathfrak{D}_{1} \oplus \mathfrak{D}_{2}$ where $\mathfrak{D}_{1}$ and $\mathfrak{D}_{2}$ are ideals of $\mathfrak{D}(\mathfrak{A})$ and that to show $\mathfrak{D}_{2}$ acts completely reducibly on $\mathfrak{A}$ it is enough to show that $\mathfrak{D}_{3}=\{$ linear transformations $D$ of $\mathfrak{N} ; D$ satisfies (5)\} acts completely reducibly on $\mathfrak{N}$.

The correspondence $R:\left.z \rightarrow R_{z}\right|_{\mathfrak{N}}$ is an associative homomorphism of 3 into the algebra of all linear transformations of $\mathfrak{N}$. The image of $R$ is a semisimple associative algebra, hence is completely reducible on $\mathfrak{N}$. Hence $\mathfrak{D}_{3}$ is completely reducible by Theorem 18, p. 102 of [6]. Hence $\mathfrak{D}(\mathfrak{U})$ acts completely reducibly on $\mathfrak{A}$.

To show the converse, we suppose that $\mathfrak{D}(\mathfrak{R})$ is completely reducible acting on $\mathfrak{A}$. Let

$$
\mathfrak{R}=\text { linear span of all }[a, n], \quad a \in \mathfrak{A}, n \in \mathfrak{N} .
$$

$\mathfrak{R}$ is invariant under each $D \in \mathfrak{D}(\mathfrak{H})$ since $[x, y] D=[x D, y]+[x, y D]$ and $\mathfrak{N}$ is characteristic in $\mathfrak{A}$ (Lemma 2 of [10]). Hence $\mathfrak{A}=\mathfrak{R} \oplus \mathfrak{J}$ where $\mathfrak{J}$ is $\mathfrak{D}(\mathfrak{U})$-invariant. If $a \in \mathfrak{I}$, then $[a, n]=a\left(R_{n}-L_{n}\right) \in \mathfrak{A} \cap \mathfrak{I}=0$ since $R_{n}-L_{n} \in \mathfrak{D}(\mathfrak{H})$. Hence $\mathfrak{R}$ is the linear span of all $[a, n], a \in \mathfrak{R}, n \in \mathfrak{N}$. Then $\mathfrak{R}=[\mathfrak{R}, \mathfrak{N}]=[[\mathfrak{R}, \mathfrak{N}], \mathfrak{N}]=\cdots$. Since $\mathfrak{R} \subseteq \mathfrak{N}$, we must have $\mathfrak{R}=0$ and each $n \in \mathfrak{N}$ commutes with each $a \in \mathfrak{A}$. Next let

$$
\mathfrak{\Omega}_{1}=\text { linear span of all }(x, z, y), \quad x, z \in \mathfrak{A}, y \in \mathfrak{N} .
$$

Then $\mathfrak{R}_{\mathbf{1}}$ is invariant under each $D \in \mathfrak{D}(\mathfrak{H})$ since

$$
(x, z, y) D=(x D, y, z)+(x, y D, z)+(x, y, z D) .
$$

Hence $\mathfrak{U}=\mathfrak{R}_{1} \oplus \mathfrak{I}_{1}$ where $\mathfrak{I}_{1}$ is $\mathfrak{D}(\mathfrak{U})$-invariant. If $z \in \mathfrak{I}_{1}$, then $(x, z, y)=$ $z\left[L_{x}, R_{y}\right] \in \mathfrak{J}_{1} \cap \Re_{1}=0$ since $\left[L_{x}, R_{y}\right] \in \mathfrak{D}(\mathfrak{R})$. Hence $\boldsymbol{\Re}_{1}$ is generated by $(x, z, y), y \in \mathfrak{N}, z \in \mathfrak{R}_{1}, x \in \mathfrak{A}$. Suppose that $x \in \mathfrak{I}_{1}$ along with $y \in \mathfrak{N}$, $z \in \mathfrak{\Omega}_{1}$. Then $(x, z, y)=(x, y, z)+(z, x, y)=(x, y, z)$. If, in addition, 
$y \in \mathfrak{I}_{1} \cap \mathfrak{N}$, then $(x, z, y)=(x, y, z)=0$. Hence $\Omega_{1}$ is generated by

$$
(x, z, y), \quad y \in \mathfrak{N}, z, x \in \mathfrak{R}_{\mathbf{1}},
$$

and

$$
(x, z, y), \quad x \in \mathfrak{I}_{1}, z, y \in \mathfrak{R}_{1} .
$$

We show $\Omega_{1}=0$ by considering the Penico chain: $\mathfrak{N}^{\langle 0\rangle}=\mathfrak{N}, \mathfrak{N}^{\langle i+1\rangle}=$ $\mathfrak{A}\left(\mathfrak{N}^{\langle i\rangle}\right)^{2}+\left(\mathfrak{N}^{\langle i\rangle}\right)^{2}$ for $i>0$. Each $\mathfrak{N}^{\langle i\rangle}$ is an ideal in $\mathfrak{A}$ and since $\mathfrak{N}$ is nilpotent, there exists a $t$ such that $\mathfrak{N}^{\langle t\rangle}=0$ (Theorem 3 of [10]). It is readily verified that each of (6) and (7) is in $\mathfrak{N}^{\langle s\rangle}$ for each $s$, hence $\mathfrak{\Omega}_{1}=0$. Since $\mathfrak{A}$ is flexible, $(y, z, x)=-(x, z, y)=0$ for all $y \in \mathfrak{N}, z, x \in \mathfrak{A}$. Then $(x, y, z)=(x, z, y)-(z, x, y)=0$ for all $y \in \mathfrak{N}, z, x \in \mathfrak{A}$. Hence $\mathfrak{R} \subseteq \mathfrak{C}$.

Next, to show that $\mathfrak{N}^{2}=0$, we suppose to the contrary that $\mathfrak{N}^{2} \neq 0$. Since $\mathfrak{N} \subseteq \mathfrak{C}, \mathfrak{C}=3+\mathfrak{N}$ where 3 is as in (3). Also $\mathfrak{C}$ is $\mathfrak{D}(\mathfrak{R})$-invariant so that $\mathfrak{D}(\mathfrak{U})$ acts completely reducibly on $\mathfrak{C}$. Consider $\mathfrak{N} \supset \mathfrak{N}^{2} \supset \cdots \supset$ $\mathfrak{N}^{k}=0(k>2)$ and note that since $\mathfrak{C}$ is associative, each $\mathfrak{N}^{i}$ is a characteristic ideal of $\mathfrak{C}$. Let $\mathfrak{I}$ be a $\mathfrak{D}(\mathfrak{U})$-invariant complement of $\mathfrak{N}^{k-1}$ and we show the existence of a derivation $D$ of $\mathfrak{C}$ which may be extended to a derivation of $\mathfrak{A}$ and is such that $\mathfrak{I}$ is not invariant under $D$. The ideas are precisely the same as those used in [4, p. 694]. Let $r \in \mathfrak{N}^{k-2}$ and define the linear transformation $D_{r}$ of $\mathfrak{C}$ by

$$
x D_{r}=r x \quad \text { if } x \in \mathfrak{N} \quad \text { and } \quad x D_{r}=0 \quad \text { if } x \in \mathcal{Z} .
$$

Then $D_{r}$ is a derivation of $\mathfrak{C}$ which can be extended to a derivation of $\mathfrak{A}$ by letting $\mathcal{S}^{\prime}+\mathfrak{B}$ be annihilated, where $\mathcal{S}^{\prime}$ and $\mathfrak{B}$ are as in (3). (See the proof of Theorem 7 of [10].) Since $\mathfrak{N}^{k-1} \neq 0$, there exists $r \in \mathfrak{N}^{k-2}$ such that $D_{r} \neq 0$. Hence there exists $x \in \mathfrak{T}$ such that $0 \neq x D_{r}=x r \in \mathfrak{N}^{k-1}$. This contradicts the $\mathfrak{D}(\mathfrak{L})$-invariance of $\mathfrak{I}$ and the result holds.

2. The alternative case. An algebra $\mathfrak{A}$ is said to be alternative if the identities

$$
x^{2} y=x(x y), \quad y x^{2}=(y x) x
$$

are satisfied in $\mathfrak{A}$. Alternative algebras have been investigated by many authors and they are discussed in detail in [9]. In particular, we use many of the results of Schafer in [8]. One of the results appearing there is that an alternative algebra over a field of characteristic 0 is semisimple if and only if its derivation algebra is semisimple or 0 . This result will be used in what follows.

Proof of THE Alternative CaSe. Assume that $\mathfrak{N} \subseteq \mathbb{C}$ and that $\mathfrak{N}^{2}=0$. Let $\mathfrak{U}=\mathfrak{N}+\mathfrak{S}$ be a Wedderburn decomposition of $\mathfrak{A}$. Then

$$
\mathfrak{s}=3 \oplus \mathfrak{S}^{\prime}
$$


where 3 is the center of $\subseteq$ and $\mathcal{S}^{\prime}$ is the commutator subspace of $\subseteq$ (Lemma 4 of [8]). As in the standard case we show that $\mathfrak{D}(\mathfrak{R})=\mathfrak{D}_{1}+\mathfrak{D}_{2}$ where

and

$$
\mathfrak{D}_{1}=\{D \in \mathfrak{D}(\mathfrak{A}) ; D: \subseteq \rightarrow \mathfrak{S}, D: \mathfrak{R} \rightarrow 0\}
$$

$$
\mathfrak{D}_{2}=\{D \in \mathfrak{D}(\mathfrak{U}) ; D: \subseteq \rightarrow 0\}
$$

and that $\mathfrak{D}_{1}$ and $\mathfrak{D}_{2}$ act completely reducibly on $\mathfrak{A}$. The result then holds as in the standard case.

Let $D \in \mathfrak{D}(\mathfrak{Q})$. Then $D$ restricted to $\subseteq$ may be extended to a derivation $\hat{D}$ of $\mathfrak{A}$ where

$$
\hat{D}=\sum\left(R_{\left[x_{i}, z_{i}\right]}-L_{\left[x_{i}, z_{i}\right]}-3\left[L_{x_{i}}, R_{z_{i}}\right]\right)
$$

where $x_{i}, z_{i} \in \mathfrak{A}$ (Theorem 5 of [8]). Since $\mathfrak{N} \subseteq \mathfrak{C}, x_{i}, z_{i}$ may be assumed to be in $\mathfrak{S}$. Hence $\hat{D} \in \mathfrak{D}_{1}$ and $D: \mathfrak{S} \rightarrow \mathfrak{S}$. Also any derivation of $\mathfrak{S}$ can be extended to an element of $\mathfrak{D}_{1}$. Hence to show that $\mathfrak{D}_{1}$ acts completely reducibly on $\mathfrak{A}$ it is enough to show that $\mathfrak{D}(\mathfrak{S})$ acts this way on $\mathfrak{S}$. But $\mathfrak{S}$ semisimple implies that $\mathfrak{D}(\mathfrak{S})$ is semisimple or 0 ; hence $\mathfrak{D}(\mathfrak{S})$ acts completely reducibly on $\subseteq$ by a result of Weyl (p. 79 of [6]).

As in the standard case, if $D \in \mathfrak{D}(\mathfrak{U})$, then $D$ restricted to $\mathfrak{N}(\mathfrak{N}$ is characteristic by Lemma 6 of [8]) commutes with all $R_{a}, a \in 3$, and conversely any such linear transformation of $\mathfrak{R}$ can be extended to an element of $\mathfrak{D}_{2}$ by first extending it to a derivation of $\mathfrak{C}=3+\mathfrak{N}$ which annihilates 3 and then to a derivation of $\mathfrak{A}$ which annihilates $\mathcal{S}^{\prime}$ by the argument in the proof of Theorem 7 of [8]. The proof is then completed as in the standard case.

For the converse, we assume $\mathfrak{D}(\mathfrak{H})$ acts completely reducibly on $\mathfrak{A}$. Let $\mathfrak{D}=$ subspace of $\mathfrak{D}(\mathfrak{H})$ spanned by all derivations of $\mathfrak{A}$ of the form $R_{[x, z]}-L_{[x, z]}-3\left[L_{x}, R_{z}\right], x \in \mathfrak{A}, z \in \mathfrak{N}$. As Schafer has shown (p. 16 of [8]), $\mathfrak{D}$ is an ideal of $\mathfrak{D}(\mathfrak{U})$. Let

$$
\mathfrak{R}=\text { linear span of all }[y,[x, z]]-3(x, y, z), \quad y, x \in \mathfrak{A}, z \in \mathfrak{R} .
$$

Note that $\mathfrak{R}=\mathfrak{H D}$. Then $\mathfrak{R}$ is $\mathfrak{D}(\mathfrak{H})$-invariant since $\mathfrak{A D}(\mathfrak{H})=(\mathfrak{H D}) \mathfrak{D}(\mathfrak{H}) \subseteq$ $\mathfrak{A D}=\mathfrak{A}$. Hence $\mathfrak{A}=\mathfrak{R} \oplus \mathfrak{I}$ where $\mathfrak{I}$ is $\mathfrak{D}(\mathfrak{U})$-invariant. Now $\mathfrak{J} \mathfrak{D} \subseteq \mathfrak{U D} \cap$ $\mathfrak{J} \mathfrak{D}(\mathfrak{H}) \subseteq \mathfrak{A} \cap \mathfrak{I}=0$ implies that $\mathfrak{R}=\mathfrak{H D}=(\mathfrak{R} \oplus \mathfrak{J}) \mathfrak{D}=\mathfrak{R D}$. Note that $\mathfrak{A}$ alternative implies that each $\mathfrak{N}_{i}$ is an ideal of $\mathfrak{U}$ where $\mathfrak{N}_{2}=\mathfrak{N} \cdot \mathfrak{N}$ and $\mathfrak{N}_{i+1}=\mathfrak{N N}_{i}+\mathfrak{N}_{i} \mathfrak{N}$ for $i>2$, and this chain must terminate at 0 . We claim $\mathfrak{R} \subseteq \mathfrak{N}_{i}$ for each $i$. For let $y \in \mathfrak{R}, x \in \mathfrak{A}, z \in \mathfrak{N}$. Then

$$
\begin{aligned}
& {[y,[x, z]]-3(x, y, z) }=[y,[x, z]]+3(y, x, z) \\
&=y(x z-z x)-(x z-z x) y+3(y x) z-3 y(x z) \in \mathfrak{N}_{2} .
\end{aligned}
$$

Hence $\Re \subseteq \mathfrak{N}_{2}$. Now if $\Re \subseteq \mathfrak{N}_{i}$, then the same computation yields that $\mathfrak{R} \subseteq \mathfrak{N}_{i+1}$. Hence $\mathfrak{R}=0$ and $\mathfrak{N} \subseteq$ nucleus of $\mathfrak{A}$ by Lemma 9 of [8]. Now 
$R_{n}-L_{n} \in \mathfrak{D}(\mathfrak{R})$ for each $n \in \mathfrak{N}$. Hence $\mathfrak{N} \subseteq \mathbb{C}$ by the same argument given in the standard case. Finally $\mathfrak{N}^{2}=0$ is now completed as in the standard case.

REMARK. The referee has noted that it is possible that the Theorem holds if $\mathfrak{A}$ is a $J$-algebra with commutators completely alternative such that $\left[L_{x}, L_{y}\right]+\left[L_{x}, R_{y}\right]+\left[R_{x}, R_{y}\right]$ is a derivation for all $x, y \in \mathfrak{A}$ (see [2]). $\mathfrak{A}$ is then a generalization of both standard and alternative algebras. It can be verified that the conditions of the Theorem are sufficient for $\mathfrak{D}(\mathfrak{U})$ to be completely reducible. The necessity of the conditions remains an open question.

\section{REFERENCES}

1. A. A. Albert, Power-associative rings, Trans. Amer. Math. Soc. 64 (1948), 552-593. MR 10, 349.

2. R. E. Block, A unification of the theories of Jordan and alternative algebras, Amer. J. Math. 94 (1972), 389-412.

3. C. Chevalley, Théorie des groupes de Lie. Tome II. Groupes algébriques, Actualités Sci. Indust., no. 1152, Hermann, Paris, 1951. MR 14, 448.

4. G. P. Hochschild, Semi-simple algebras and generalized derivations, Amer. J. Math. 64 (1942), 677-694. MR 4, 71.

5. N. Jacobson, Completely reducible Lie algebras of linear transformations, Proc. Amer. Math. Soc. 2 (1951), 105-113. MR 14, 241.

6. - Lie algebras, Interscience Tracts in Pure and Appl. Math., no. 10, Interscience, New York, 1962. MR 26 \#1345.

7. E. Kleinfeld, Standard and accessible rings, Canad. J. Math. 8 (1956), 335-340. MR 17, 1180.

8. R. D. Schafer, Representations of alternative algebras, Trans. Amer. Math. Soc. 72 (1952), 1-17. MR 13, 527.

9. - An introduction to non-associative algebras, Pure and Appl. Math., vol. 22, Academic Press, New York, 1966. MR 35 \#1643.

10. — Standard algebras, Pacific J. Math. 29 (1969), 203-223. MR 39 \#5647.

Department of Mathematics, North Carolina State University, Raleigh, North Carolina 27607 